\title{
The Effect of 16-Week Plyometric Training on Explosive Actions in Early to Mid-Puberty Elite Soccer Players
}

\author{
Quirin Söhnlein, ${ }^{1,2}$ Erich Müller, ${ }^{1}$ AND Thomas L. StögGL ${ }^{1,3}$ \\ ${ }^{1}$ Department of Sport Sciences and Kinesiology, Paris-Lodron University of Salzburg, Salzburg, Austria; ${ }^{2}$ Red Bull Soccer \\ Academy West Africa Ltd., Sogakope, Ghana; and ${ }^{3}$ Department of Health Sciences, Swedish Winter Sports Research Center, \\ Mid Szeden University, Östersund, Sweden
}

\begin{abstract}
Söhnlein, Q, Müller, E, and Stöggl, TL. The effect of 16-week plyometric training on explosive actions in early to mid-puberty elite soccer players. J Strength Cond Res 28(8): 2105-2114, 2014-Plyometric training (PT) programs are widely used to improve explosive actions in soccer players of various ages, although there is debate about optimal training duration and time course of improvement. Twenty-two early to mid-puberty elite soccer players were assigned to a control group (CG, $n=10$, regular soccer training) or a plyometric training group (PTG, $n=12$, regular soccer training substituted with 2 PT sessions each week). Both groups trained for 16 weeks during the in-season period. Control group performed only tests at baseline and after intervention, whereas PTG performed additional tests after 4,8 , and 12 weeks. During each test, subjects' performances in speed (10 and $30 \mathrm{~m} ; 5$ and $20 \mathrm{~m}$ ), agility, shuttle run, multiple 5 bounds (MB5), and standing long jump (LJ) were recorded. The PTG showed improved performance in 20-m sprint time $(-3.2 \%)$, agility time $(-6.1 \%)$, MB5 distance (+11.8\%), and $\mathrm{LJ}$ distance $(+7.3 \%$ ) (all, $p \leq 0.05$ ) after 16 weeks. All these improvements were higher compared with CG (all, $p \leq 0.05$ ). The time course of improvement in the PT group showed that $20-\mathrm{m}$ sprint time improved after 16 weeks $(p=0.012)$; agility after $4(p=0.047)$ and 8 weeks ( $p=0.004)$ but stopped after 12 weeks $(p=0.007)$; MB5 after $8(p=0.039), 12(p=0.028)$, and 16 weeks $(p<0.001)$; and $L J$ improved after $4(p=0.045), 12(p=0.008)$, and 16 weeks $(p<0.001)$. Plyometric training seems to be an appropriate training tool to enhance some but not all explosive actions. The results indicate that the duration of a PT program is highly dependent on what type of explosive actions should be
\end{abstract}

Address correspondence to: Dr. Thomas L. Stöggl, thomas.stoeggl@sbg. ac.at.

$28(8) / 2105-2114$

Journal of Strength and Conditioning Research

(c) 2014 National Strength and Conditioning Association improved, or whether several explosive actions should be improved at the same time.

KEY WoRDS agility, Ghanaian soccer players, program duration, sprint performance, time course

\section{INTRODUCTION}

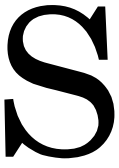

occer is rich in terms of explosive actions, demonstrating more than 700 turns (4), 30-40 sprints, and 30-40 tackles and jumps (24) during a match of professional adults. Furthermore, on average, short sprints occur every 90 seconds, lasting for 2-4 seconds each $(2,26)$, with most of the sprints starting with a sudden change in running direction or from slow running, and for the most part are no longer than a distance of $30 \mathrm{~m}(39,41)$. Although these explosive actions represent only a small percentage of a total match's minutes, they seem to be crucial for success (30). As a consequence, it has been recommended that soccer players receive independent training to be able meet these physical demands (30). Although, as mentioned above, these results are based on research of adult professional soccer players, the ability to perform explosive actions needs to be developed in youth soccer players, as well.

Almost every explosive action during soccer (e.g., sprint, change of direction, or jump) involves a stretch-shortening cycle (SSC) (23). During the SSC, the preactivated muscle is first stretched (eccentric action) and followed by the shortening (concentric) action. Plyometric exercise is a major tool for application and training of the SSC mechanism (28). Essential for the SSC is the rate of musculotendinous stretch to plyometric exercise $(5,6,18)$. The SSC stimulus provided by plyometric training (PT) programs seems to improve jumping ability $(12,13)$, agility $(22,38)$, maximal and explosive strength $(23,34)$, and sport-specific performance (35) consistently. Therefore, PT seems to be an adequate training mode to improve major soccer-specific qualities (e.g., initial acceleration, maximum velocity, and agility) (21-23,33).

Lately, in youth soccer players, PT has received a great deal of attention because it is seen as an appropriate training 
tool for improving explosive actions of young soccer players ranging from prepubertal to late pubertal ages $(7,12,21$ $23,31,38)$. Reasons for the implementation of PT in prepubertal soccer players are based on the lack of ability for building muscle mass (associated with the testosterone development in this age group) (23) and PT's advantage of forcing mainly neural adaptations (1).

For coaches and strength and conditioning professionals, it is very important to know specific program duration to improve a certain explosive action. However, there is only 1 general recommendation, regardless of the type of sport, program, and duration of PT, concerning improvements in sprint performance. This recommendation is as follows: a volume of less than 10 weeks with more than 18 sessions using high intensities and more than 80 jumps per session. It is worth noting that this meta-analysis mixed prepubertal, adolescent, and adult athletes (33). Vertical jump performance improvements are reported to appear as quickly as after 4 weeks of a PT program in adult athletes (29). For agility performance and for young athletes in general, no recommendations are available.

Therefore, the aim of this investigation was to give a recommendation about the program duration in early to mid-puberty soccer players regarding the short-term effects of PT for improving sprint performance, agility, and jumping parameters. Furthermore, this study is not only the first to report performance and training data, testosterone values, and bone age data of early to mid-puberty Ghanaian elite soccer players, but also is the first to examine the effect of 16 weeks of PT on 2 different agility tests.

\section{Methods}

\section{Experimental Approach to the Problem}

This study examined whether traditional soccer training, substituted twice a week with PT sessions, improves explosive actions of early to mid-puberty soccer players more than traditional soccer training alone. A program duration of 16 weeks was chosen to include all previous studies conducted in youth soccer players. Additionally, the study's goal was to determine the time course of improvement by measuring dependent variables every 4 weeks over a 16-week period (pretraining test, tests after 4, 8, 12, and 16 weeks). A nonrandomized 2-group repeated-measures experimental design was used. The U13 Team of a local elite academy was assigned to the control group (CG) that involved only regular in-season soccer training; the U15 Team was assigned to the experimental group (plyometric training group [PTG]), which involved a PT protocol that was integrated into the regular in-season soccer training. One week before baseline testing, bone age and testosterone values were measured to provide an indication of the biological status of the subjects. Additionally, familiarization with the testing procedure was executed. Both groups were familiar and skilled with jumping techniques because of a stabilization program performed during the last 0.5 years before the study. Baseline and postintervention testing included speed (30-m sprint, 20-m sprint), agility, shuttle run (SR5 $\times 10 \mathrm{~m}$ ), and jumping ability (multiple 5 bounds, standing long jump [LJ]). All tests were measured 48 hours after the last training session or game at the same time (7:30-9:30 AM) and under the same conditions $\left(29-33^{\circ} \mathrm{C}\right.$, no wind). All participants were encouraged to follow the same diet pattern before testing. Throughout all tests, water was consumed adlibitum.

\section{Subjects}

Twenty-nine healthy players from an elite academy in Ghana (age range, 11.2-14.7 years) were recruited; physical characteristics are presented in Table 1. All participants organized soccer for more than 4 years on a regular basis $(3-5$ times per week) and were at least 0.5 years in the academy. Because of organizational limitations in this academy, no randomization of groups was possible. Therefore, the U13 Team ( $n=11$ players; age: $12.0 \pm 1.0$ years) was used as CG to supervise the effects of regular soccer training, and the U15 Team ( $n=18$ players; age: $13.0 \pm 0.8$ years) was used as PTG. Six players of PTG dropped out, 2 because of missing tests after 4 and 8 weeks, 1 left the academy before the study was finished, and 3 missed more than $20 \%$ of all PT sessions due to injuries received in games. All participants, parents, or guardians were informed prior in detail about the testing and training procedure, as well as possible benefits and risks of the study, and written informed consent was provided. The study was approved by the institutional board, and procedures were in accordance with the Helsinki declaration.

\section{Procedures}

Plyometric Training Protocol. Before every PT session, a standardized warm-up was completed as follows: running, very simple coordination exercises, preparation jumps with the main focus on stabilization, core exercises, and dynamic stretching. The duration for every warm-up was 8-12 minutes. Each PT session lasted 20-35 minutes, and every first session of the following week was performed 48 hours after the game

TABLE 1. Subjects' physical characteristics.*

\begin{tabular}{lcc}
\hline & CG $(n=11)$ PTG $(n=12)$ \\
\hline Age $(\mathrm{y})$ & $12.3 \pm 0.8$ & $13.0 \pm 0.9 \dagger$ \\
Bone age $(\mathrm{y})$ & $13.1 \pm 0.9$ & $14.0 \pm 1.3$ \\
Testosterone & $404 \pm 90$ & $445 \pm 58$ \\
$\left(\mathrm{ng} \cdot \mathrm{dl}^{-1}\right)$ & & \\
Height $(\mathrm{cm})$ & $154.2 \pm 5.8$ & $162.4 \pm 9.5 \ddagger$ \\
Weight $(\mathrm{kg})$ & $40.8 \pm 4.5$ & $51.0 \pm 6.4 \S$ \\
\hline & \\
& \\
&
\end{tabular}


on weekends (games were mostly on Saturday mornings; therefore, PT started mostly on Monday morning). The second PT session was completed 72 hours after the first PT session (most of the time on Thursday mornings) (9). Every first PT session in each week focused on improving the vertical-horizontal leap, whereas every second session focused on improving lateral jumping ability. Plyometric exercises apart from ladder drills were kept constant to give a clear indication of improvement. To ensure an appropriate training intensity for children and limited stress on musculotendon units during the plyometric exercise, volume and intensity were increased progressively (28). During every verticalhorizontal PT session, the following jumps were performed: 2-footed ankle hop forward, hurdle jumps (with height increasing during the 16 weeks), single leg hop forward, and squat jump. During every lateral PT session, the following jumps were performed: lateral bound stabilization, lateral hurdle jumps, double leg zigzag, and single leg hop lateral. Both sessions consisted of a volume of 2-5 sets and 6-16 repetitions. The total number of ground contacts per week started at 112 during the first week, and gradually increased to 350 after 16 weeks. After each PT session, participants had to pass ladder drills with the goal of learning at least 1 new movement every session. All ladder drills were first performed qualitatively to ensure the right movement pattern and then focused on speed in all kinds of variations, with each drill lasting for about 3-6 seconds and a total duration of 2-5 minutes. Plyometric exercises were executed at maximal intensity. Furthermore, all PT sessions were completed on an artificial turf pitch to minimize first landing impact and to be as soccer-specific as possible. Throughout every session, proper technique was ensured through verbal cues and demonstration by the researchers.

Soccer Training Protocol. Both groups participated in the regular soccer training over 16 weeks. Soccer training included training in fast footwork, technical skills and moves (easy/difficult), position games (small/big), 1 vs. 1 offensive and 1 vs. 1 defensive, and tactical games with various objectives. Total training data are presented in Table 2.

General Testing Procedure. Warm-up was standardized for all tests as follows: 5-minute running, 10-minute strength and flexibility exercises using their own body weight, 2-minute dynamic stretching, and 5-minute sprint-specific warm up. All tests were separated by a 5-10 minutes of break. Each participant had 2 trials for each test; the best performance of each test was recorded. All tests were performed outdoors on an artificial turf pitch with participants wearing their regular training kit and soccer boots in the following order.

Sprint Testing. For measurement of sprint performance, $30-\mathrm{m}$ and a $20-\mathrm{m}$ sprint tests were used. The $30-\mathrm{m}$ sprint test was measured with a $10-\mathrm{m}$ split time; the $20-\mathrm{m}$ sprint test was measured with a 5-m split time for the determination of
TABLE 2. Subjects' training characteristics after 16 weeks.*

\begin{tabular}{lcc}
\hline & CG $(n=10)$ & $\mathrm{PTG}(n=12)$ \\
\hline Number of trainings & $72 \pm 1$ & $71 \pm 6$ \\
Number of games & $12 \pm 0$ & $11 \pm 2 \dagger$ \\
Total (min) & $6,805 \pm 230$ & $6,757 \pm 530$ \\
Total FS (min) & $5,684 \pm 165$ & $4,797 \pm 412$ \\
Total GT (min) & $669 \pm 74$ & $550 \pm 112$ \\
Total Core (min) & $453 \pm 29$ & $414 \pm 23$ \\
Total PLY (min) & $0 \pm 0$ & $997 \pm 65 \ddagger$ \\
\hline
\end{tabular}

${ }^{*} \mathrm{CG}=$ control group; PTG = plyometric training group; $F S=$ football skills, technical and tactical training; GT = game time; Core = core and stabilization training; $P L Y=$ plyometric training. Data are presented as mean $\pm S D$.

$\dagger p \leq 0.05$, significant difference between groups.

$\ddagger p<0.001$, significant difference between groups.

starting acceleration. Participants started $0.3 \mathrm{~m}$ before the first infrared photoelectric gate (Brower Timing Systems, Draper, UT, USA), which were placed $0.75 \mathrm{~m}$ above the ground to ensure a capture of the trunk movement and avoid false signals by limb motion. In total, 3 single beam photoelectric gates were used for both the $20-\mathrm{m}$ and $30-\mathrm{m}$ sprint. Current research on sprint ability in preadolescent and adolescent subjects displayed intraclass correlation coefficients of 0.88 $0.98(32)$.

Change of Direction Testing. The hurdle agility run (HAR) was performed as previously described by Gonaus and Müller (14). Participants started $0.3 \mathrm{~m}$ behind the starting line and then completed the agility course involving jumps over hurdles and $90^{\circ}$ turns. Time to finish the HAR was recorded by infrared photoelectric cells (Brower Timing Systems) placed $0.75 \mathrm{~m}$ above the ground. Intraclass correlation coefficient was reported to be acceptable with the values of 0.74-0.79 (15).

A $5 \times 10-m$ Shuttle Run. This test was used to evaluate each participant's soccer-specific speed and agility. In this running and turning test, players had to complete 5 runs of $10 \mathrm{~m}$ at maximal speed. Turns had to be as quick as possible with 1 foot-freely selectable-crossing a line while changing direction $(14,40)$. Time was recorded by infrared photoelectric gates (Brower Timing Systems) placed at the starting line and the finish line. Intraclass correlation coefficient was reported to be moderate to high, 0.81-0.97 (15).

Multiple 5 Bounds Test. From a standing position with both feet on the ground, participants tried to cover as much distance as possible with 5 forward jumps by alternating left and right leg contacts. This test has been recommended for the measurement of lower-limb muscle power instead of the 
vertical jump test and is considered to be soccer specific (12). The covered distance was measured to the nearest $1 \mathrm{~cm}$ using a tape measure. The coefficient of variation for testretest trials is reported with $2.7 \%$ (23).

Standing Long Jump. From a starting position with their feet at shoulders' width behind a line marked on the ground and their hands in a neutral position, participants executed a countermovement with their legs and their arms and jumped in a horizontal direction. Participants had to land with both feet at the same time and were not allowed to fall forward or backward. The horizontal distance between the starting line and the heel of the rear foot was recorded through tape measure to the nearest $1 \mathrm{~cm}$ (19). The coefficient of variation for test-retest trials is reported with $2.6 \%(23)$.

Bone Age Measurement. Skeletal maturation was determined with an x-ray of the left hand and wrist under full body protection against radioactivity. Bone age was examined by KGS 4.5 Software (Pharmacia \& Upjohn, Kalamazoo, MI, USA). Ossification center and ossification of the wrist were optically compared (37), and together with values for height, weight, and date of birth were inscribed into the software for the calculation of bone age.

Testosterone Measurement. Blood samples were drawn through venipuncture from an antecubital arm vein using a safety butterfly set equipment for children, with subjects always in a semirecumbent position. All blood samples were taken in the morning from 7:30 to 8:30 hours. Blood was collected into vacutainer tubes containing SST-Gel and clot activator. Blood was allowed to clot at room temperature $\left(23^{\circ} \mathrm{C}\right)$ for 20 minutes and subsequently centrifuged $\left(1,500 \mathrm{~g}, 4^{\circ} \mathrm{C}, 15\right.$ minutes) for serum separation (23). The resulting serum was used for testosterone measurement. Blood samples were analyzed within 45 minutes after being centrifuged. Testosterone levels were analyzed by immunoassay analysis system, i-chroma testosterone test (Boditech Med Inc., Chuncheon, Korea). Therefore, detection buffer was taken from refrigerator and left at room temperature for 20 minutes. Further, a $75 \mu \mathrm{L}$ of sample serum was added to the tube containing detection buffer and mixed. Seventy-five microliter of this mixture were taken within 30 seconds and transferred onto the test device. After exactly 15 minutes, the testing device was inserted into the i-chroma testosterone machine and analyzed. Results were written down as nanogram per milliliter, and further recalculated to nanogram per deciliter.

\section{Statistical Analyses}

Data are presented as mean $\pm S D$. Data were checked for normal distribution with the Shapiro-Wilk's test. Two-way repeated-measures analysis of variance (ANOVA) (Time $\times$ Group) was applied to test for main effects between baseline and postintervention testing and between the 2 groups (PTG vs. CG). Independent sample $t$-tests were used to determine the differences between PTG and CG at baseline. Within- subject effects for both groups were analyzed using paired sample $t$-tests. Time course of changes in PTG was investigated by an ANOVA with repeated measures and Bonferroni's post hoc analysis for all variables revealing significant time effects in the $2 \times 2$ ANOVA. In addition, the values obtained were evaluated further by calculating the effect size $\left(\eta^{2}\right)$ and statistical power. The effect sizes were classified as trivial $(<0.01)$, small $(0.01-0.06)$, moderate $(0.06-0.14)$, and large $(>0.14)(10)$. The level of significance was set at $\alpha=$ 0.05 . For all statistical tests, PASW statistic version 21.0 (SPSS Inc., Chicago, IL, USA) was used.

\section{Results}

\section{Effects of 16-Week Plyometric Training}

Values for all variables at baseline and after intervention are presented in Table 3. A main effect for time was found for all tests with the exception of the $5 \times 10$-m shuttle run (SR). Time $\times$ group interaction effects were found for agility time, multiple 5 bounds (MB5) distance, and LJ distance (all $p \leq$ $0.05)$, with a trend for $20-\mathrm{m}$ sprint time $(p<0.1)$, indicating more pronounced performance improvement in PTG when compared with CG. Control group demonstrated improvements in 20-m sprint time ( $p<0.01), 30$-m sprint time, and MB5 distance (both, $p \leq 0.05$ ). Plyometric training group revealed performance improvements within all tests with the exception of 10 -m sprint and $5 \times 10$ - $\mathrm{m} \mathrm{SR}$ performance. All significant effects are classified as large.

\section{Time Course of Improvement}

Within PTG, large time effects for 20-m sprint time $\left(F_{(4,44)}=\right.$ $6.80, p<0.001,{ }_{p} \eta^{2}=0.38$, power $\left.=0.99\right)$, HAR performance $\left(F_{(4,44)}=12.66, p<0.001,{ }_{p} \eta^{2}=0.54\right.$, power $\left.=1.00\right)$, MB5 distance $\left(F_{(4,44)}=25.87, p<0.001,{ }_{p} \eta^{2}=0.70\right.$, power $\left.=1.00\right)$, and $L J$ distance $\left(F_{(4,44)}=13.64, p<0.001, p^{2}=0.72\right.$, power $=$ $1.00)$ were found. Further post hoc analysis revealed improvement in 20-m sprint after 16 weeks only $(p=0.012)$ (Figure $1 \mathrm{~A}$ ). When compared with baseline performance change of direction, performance (HAR) was improved after $4(p=0.047), 12$ $(p=0.004)$, and 16 weeks $(p=0.007)$ (Figure 1B).

Multiple 5 bounds performance was improved after $8(p=$ $0.039), 12(p=0.028)$, and 16 weeks $(p<0.000)$ when compared with baseline values. Improvements were also found between week 4 and week $16(p<0.001)$, week 8 and week $16(p<0.001)$, and week 12 and week $16(p<$ 0.001) (Figure 2A). Long jump performance was improved in PTG after $4(p=0.045), 12(p=0.008)$, and 16 weeks $(p<$ 0.001 ) when compared with baseline values (Figure 2B). Furthermore, improvements were also found between week 4 and week $16(p=0.044)$, and week 8 and week 16 $(p=0.022)$.

\section{Correlations}

No significant correlations between chronological age and bone age were detected $(r=0.36, p>0.05)$. Bone age was associated with $10-\mathrm{m}, 30-\mathrm{m}(r=-0.72 ; r=-0.66$, both $p<$ 
TABLE 3. Effect of 16-week plyometric training on explosive actions.*

\begin{tabular}{|c|c|c|c|c|c|c|c|}
\hline & CG $(n$ & $=10)$ & PTG ( & $(n=12)$ & & ANOVA & \\
\hline & Baseline & After training & Baseline & After training & $P$ value & $\begin{array}{l}\text { Effect size } \\
\qquad\left({ }_{p} \eta^{2}\right)\end{array}$ & $\begin{array}{l}\text { Test } \\
\text { power }\end{array}$ \\
\hline 10-m sprint $(\mathrm{s})$ & $1.87 \pm 0.08$ & $1.86 \pm 0.07$ & $1.79 \pm 0.06 \dagger$ & $1.75 \pm 0.06 \dagger$ & $\begin{array}{l}\leq 0.05 \ddagger \\
<0.01 \S \\
\leq 0.05\end{array}$ & $\begin{array}{l}0.23 \\
0.37 \\
0.22\end{array}$ & $\begin{array}{l}0.65 \\
0.91 \\
0.61\end{array}$ \\
\hline 30-m sprint (s) & $4.58 \pm 0.15$ & $4.49 \pm 0.18 \pi$ & $4.38 \pm 0.18 \dagger$ & $4.27 \pm 0.13 \dagger \#$ & $\begin{array}{l}<0.001 \ddagger \\
<0.01 \S \\
\mathrm{NS} \|\end{array}$ & $\begin{array}{l}0.49 \\
0.34\end{array}$ & $\begin{array}{l}0.99 \\
0.86\end{array}$ \\
\hline 5-m sprint (s) & $1.10 \pm 0.05$ & $1.08 \pm 0.03$ & $1.06 \pm 0.04$ & $1.02 \pm 0.04 \pi \dagger$ & $\begin{array}{l}<0.01 \ddagger \\
<0.01 \S \\
\mathrm{NS} \|\end{array}$ & $\begin{array}{l}0.30 \\
0.30\end{array}$ & $\begin{array}{l}0.80 \\
0.79\end{array}$ \\
\hline 20-m sprint (s) & $3.31 \pm 0.10$ & $3.26 \pm 0.11 \#$ & $3.15 \pm 0.14 \dagger$ & $3.05 \pm 0.10 \uparrow^{\star \star}$ & $\begin{array}{l}<0.001 \ddagger \\
<0.01 \S \\
<0.1 \|\end{array}$ & $\begin{array}{l}0.58 \\
0.44 \\
0.14\end{array}$ & $\begin{array}{l}1.00 \\
0.97 \\
0.39\end{array}$ \\
\hline $\operatorname{HAR}(\mathrm{s})$ & $11.89 \pm 0.57$ & $11.71 \pm 0.61$ & $11.66 \pm 0.79$ & $10.95 \pm 0.58 \dagger^{\star *}$ & $\begin{array}{l}<0.01 \ddagger \\
\text { NSS } \\
\leq 0.05\end{array}$ & $\begin{array}{l}0.59 \\
0.22\end{array}$ & $\begin{array}{l}0.97 \\
0.61\end{array}$ \\
\hline $\mathrm{SR} 5 \times 10 \mathrm{~m}(\mathrm{~s})$ & $11.87 \pm 0.24$ & $11.81 \pm 0.21$ & $11.53 \pm 0.26 \dagger$ & $11.40 \pm 0.33 \dagger$ & $\begin{array}{l}\text { NS } \ddagger \\
<0.001 \S \\
N S \|\end{array}$ & 0.43 & 0.96 \\
\hline MB5 (m) & $10.31 \pm 0.84$ & $10.82 \pm 0.89 \rrbracket$ & $11.24 \pm 0.94 \dagger$ & $12.57 \pm 0.78 \uparrow^{\star \star}$ & $\begin{array}{l}<0.001 \ddagger \\
<0.001 \S \\
<0.001\end{array}$ & $\begin{array}{l}0.81 \\
0.41 \\
0.46\end{array}$ & $\begin{array}{l}1.00 \\
0.95 \\
0.98\end{array}$ \\
\hline$L J(m)$ & $2.15 \pm 0.10$ & $2.20 \pm 0.13$ & $2.32 \pm 0.16 \dagger$ & $2.49 \pm 0.14 \dagger^{* *}$ & $\begin{array}{l}<0.001 \ddagger \\
<0.001 \S \\
\leq 0.05\end{array}$ & $\begin{array}{l}0.55 \\
0.47 \\
0.24\end{array}$ & $\begin{array}{l}1.00 \\
0.98 \\
0.67\end{array}$ \\
\hline
\end{tabular}

*Data are presented as mean $\pm S D . C G=$ control group; PTG = plyometric training group; HAR, hurdle agility run; SR5 $\times 10$ $\mathrm{m}=5 \times 10-\mathrm{m}$ shuttle run; MB5 = multiple 5 bounds test; $\mathrm{LJ}=$ standing long jump; $\mathrm{NS}=$ not statistically significant. $F$ and $P$ values were obtained by two-way ANOVA $(2 \times 2$ : group [PTG vs. CG] $\times$ time [baseline vs. after intervention]).

$\dagger p \leq 0.05$, significant difference between groups.

tMain effect: time.

§Main effect: group.

$\|$ Interaction effect: time $\times$ group.

I $p \leq 0.05$, significant difference within groups between baseline and postintervention test values.

$\# p \leq 0.01$, significant difference within groups between baseline and postintervention test values.

${ }^{\star \star} p \leq 0.001$, significant difference within groups between baseline and postintervention test values.

$0.001)$ and $20-\mathrm{m}(r=-0.57, p<0.01)$ sprint time, MB5, and LJ distance $(r=0.55 ; r=0.61$, both $p<0.01)$, demonstrating higher correlation coefficients when compared with chronological age $(r=0.43$ to 0.49 , all $p \leq 0.05)$. Testosterone levels were exclusively related to sprint performance $(r=-0.43$ to -0.48 , all $p \leq 0.05$ ). No relations of chronological age, bone age, and testosterone levels to the delta changes in performance across the 16-week training period were found.

\section{Discussion}

The main finding of this study is that PT seems to be a more appropriate stimulus to improve jumping parameters, second acceleration phase in sprint performance, and general agility compared with normal soccer training.

Together with the LJ test, the MB5 is often used as a measure of lower-limb power and coordination (12). In addition, the MB5 offers a higher specificity for soccer play- ers when compared with vertical jump tests (22). Our results demonstrated an 11.8\% improvement in MB5 after 16 weeks of PT, which was in line with the findings of Michailidis et al. (23). It was demonstrated that MB5 performance increases after 8 weeks and shows its highest increase after 16 weeks. Meylan and Malatesta (2009) demonstrated that improvements in MB5 are related to increased development of reactive strength $(r=0.66)$. A novel finding of this study is that soccer training alone seems to be a sufficient stimulus to enhance MB5 performance (4.9\% increase after 16 weeks in CG); however, the improvements were more pronounced in PTG. LJ performance was improved 7.3\% after the 16 weeks of PT, with improvements appearing as early as after 4 weeks, which is in contrast to the findings of Michailidis et al. (23) and Ingle et al. (19), who indicated no improvement in LJ after PT. This aspect might be based on the differences in the PT program applied in this study 

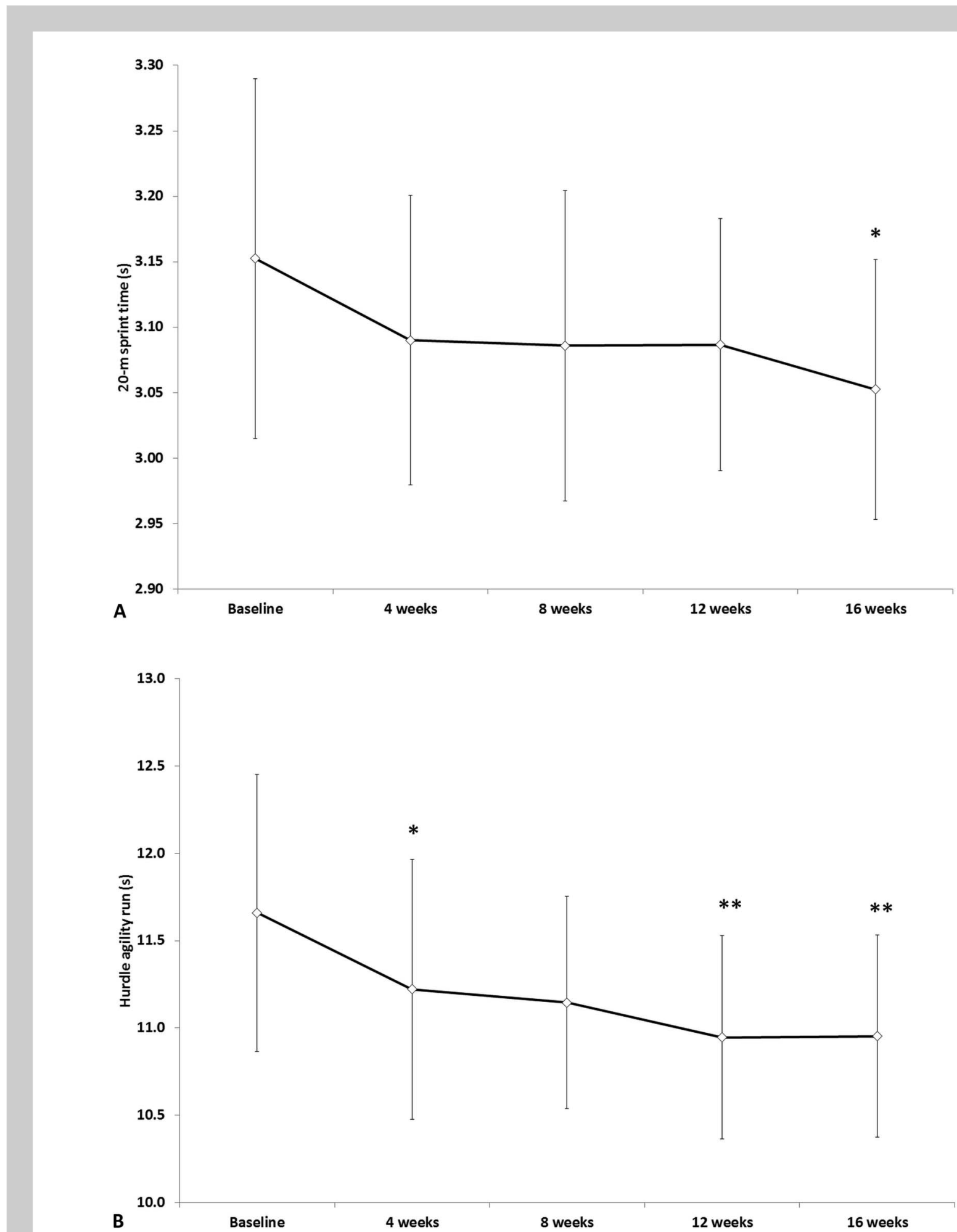

Figure 1. Time course of improvement in $20-\mathrm{m}$ sprint (A) and agility performance (B) in the plyometric training group. ${ }^{*} p \leq 0.05,{ }^{* *} p<0.001$, significantly different from pretraining values. 

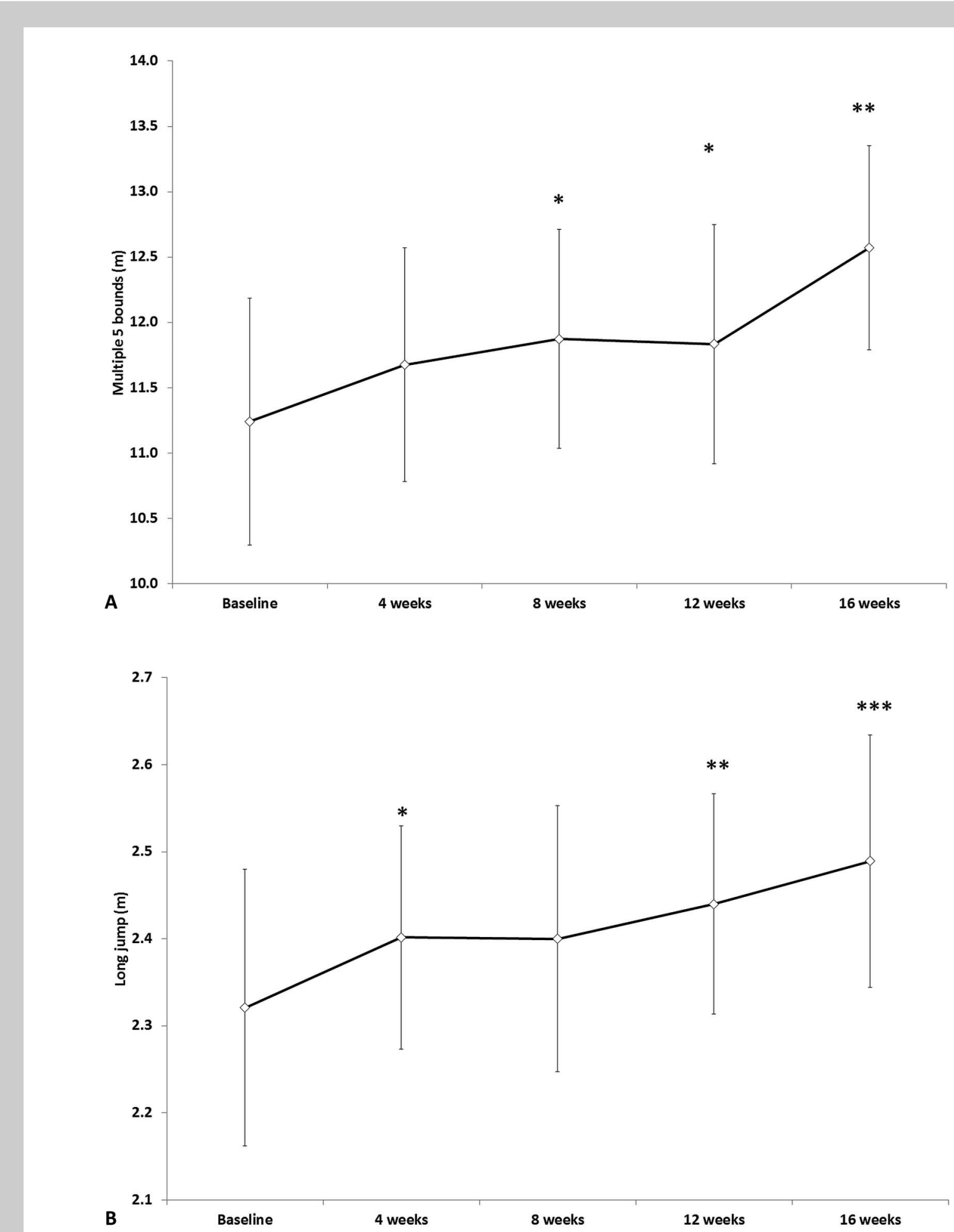

Figure 2. Time course of improvement in jumping performance with the multiple 5 bounds test $(A)$ and the standing long jump test (B) in the plyometric training group. ${ }^{*} p \leq 0.05,{ }^{* *} p<0.01,{ }^{* * *} p<0.001$, significantly different from pretraining values. 
compared with the studies above. The standing LJ involves propulsion in upward and forward directions, a motion that was provided by our PT program (e.g., hurdle jumps). It might be speculated that the marked improvement in both jumping tests, indicate that essential components of the SSC as the speed of contraction and ground contact time, were effectively enhanced by the applied PT.

This study is the first to investigate the effects of 16-week PT on sprint performance in soccer players and the time course of changes after $4,8,12$, and 16 weeks. Plyometric training is said to be an effective training method for improving sprint performance, and therefore, is relevant for sports that include sprint-type movement. If PT is applied adequately, training gains are independent of the fitness level (33). Moreover, it is known that slow SSC (longresponse) plyometrics ( $>0.25$ seconds), such as countermovement or squat jumps, transfer most directly to start and acceleration performance, whereas fast SSC (shortresponse) plyometrics $(<0.25$ seconds), such as drop jumps, have more transfer to maximum running velocity (27). Delecluse et al. (11) suggested that sprint performance is characterized by 3 phases: (a) initial acceleration phase $(0-10 \mathrm{~m}),(\mathrm{b})$ secondary acceleration phase $(10-30 \mathrm{~m})$, and (c) maximal velocity phase (after $30 \mathrm{~m}$ ). The duration of the second and third phases is highly dependent on gender, age, and performance level. Women develop maximal velocity at $25-35 \mathrm{~m}$, elite sprinters after $60 \mathrm{~m}$, whereas untrained prepubertal boys at $20-30 \mathrm{~m}$ (while initial and secondary acceleration phases are seen at $0-10 \mathrm{~m}$ and 10 $20 \mathrm{~m}$, respectively) (11,21). Acceleration (especially during the initial phase) and agility are seen as independent predictors of soccer performance during childhood and adulthood (30). This study showed improvements in 5-m time $(-3.8 \%), 10-\mathrm{m}$ time $(-2.2 \%), 20-\mathrm{m}$ time $(-3.2 \%)$, and $30-\mathrm{m}$ time $(-2.5 \%)$ in PTG, whereas only for the $20-\mathrm{m}$ sprint time was a trend found for greater improvement when compared with CG. Hence, only the second acceleration/ maximal velocity phase improved significantly, which is in line with the findings of Buchheit et al. (7), Diallo et al. (12), and Kotzamanidis (21), suggesting that it is more difficult to improve initial acceleration phase than maximal running velocity in early pubertal boys. Conversely, Michailidis et al. (23) and Meylan and Malatesta (22) reported about marked improvement in initial acceleration time and maximal velocity phase after PT, and Thomas et al. (38) found no improvement in any of the sprint phases after PT. These contradictory results might be based on the differences in frequency, volume, and intensity of PT between these studies. The results of our study cannot be attributed to the differences in the participants' conditioning levels, as suggested by Michailidis et al. (23), and is, therefore, in line with Saez de Villarreal et al. (33). Finally, the improvements in $20-\mathrm{m}$ and $30-\mathrm{m}$ sprint times in CG in this study are in line with the previously published findings of Michailidis et al. (23), which suggest that soccer training alone might contribute to speed development, probably because of its high frequency in short sprints, incorporated in most football trainings.

Next to initial acceleration speed, agility is an independent predictor of soccer performance in childhood and adulthood (30) and is defined as "a rapid whole body movement with change of velocity or direction in response to a stimulus" (36). Previous studies in early pubertal and pubertal soccer players used agility tests ranging from 5 to 19 seconds $(8,14,22,23,38)$; but those examining the effect of PT on agility performance constantly showed a marked improvement in agility performance $(22,23,38)$. A novel aspect of this study is the examination of 16 weeks of PT on 2 different change of direction (agility) tests, HAR and $5 \times 10$-m shuttle run $(\mathrm{SR} 5 \times 10-\mathrm{m})$. The first test, HAR, is considered to be a general, but a sophisticated agility test (14), whereas the second test, SR $5 \times 10-\mathrm{m}$, is considered to evaluate the soccer-specific speed and agility in its combination and is suggested as a main predictor for future drafts to the youth national teams of Austria (14). Sixteen weeks of PT revealed performance improvement in HAR $(-6.1 \%)$, whereas no changes were found for SR $5 \times 10-\mathrm{m}$. Our PT contained numerous powerful lateral movements that may have enhanced the neuromuscular adaptations that enable the athlete to switch between deceleration and acceleration movements (17), and which can be regarded as essential for HAR performance. The lack of improvement in SR $5 \times$ $10-\mathrm{m}$ might be based on the specificity of this test, including five $10-\mathrm{m}$ straight line sprints with four $180^{\circ}$ turns and a high metabolic effort. Because change of direction ( 0.4 seconds) $(3,16)$ is closely linked to the typical movement time of a countermovement jump $(0.5$ seconds; associated with slow SSC) (20), it is possible that our PT did not provide the correct stimulus; only 2 of 8 plyometric exercises within the current PT program were considered to stimulate slow SSC (squat jump and lateral bound stabilization). Furthermore, eccentric knee flexor strength seems to be more related to change of direction performance than eccentric quadriceps strength (20). Although, to the best of our knowledge, there is no published study that has examined the effect of PT on eccentric knee flexor strength, unfortunately, whether our PT provided a sufficient stimulus to improve eccentric knee flexor strength also remains questionable. Another aspect might be that our PT did not provide suffcient metabolic stimulus because SR $5 \times 10$-m is very exhausting $(>10$ seconds vs. plyometric exercise 5-8 seconds). However, our result is in line with Buchheit et al. (7), who reported that PT had no effect on repeated sprint ability tested with SRs (similar to ours). In conclusion, it seems that our PT shows improvement in HAR (a more general agility test), but is lacking in the ability to show improvement in soccer-specific speed and agility performance, as measured in SR5 $\times 10-\mathrm{m}$, although the reason why still remains unanswered.

One of the main aims of this study was to give recommendations about the program duration of a short-

2112 Journal of Strength and Conditioning Research 
term PT program in young elite soccer players to improve soccer relevant explosive actions. A novel aspect of this study is its program duration and the analysis of the time course of improvement through tests every 4 weeks. Concerning the development of jump performance, MB5 performance increases after only 8 weeks and shows its highest increase after 16 weeks; LJ improves after 4 weeks, continues to improve after 12 weeks, and results in its peak after 16 weeks. These results indicate, again, that our PT program was well structured and well performed because the highest improvements are shown after 16 weeks with no drop in between. The time course of improvement in $20-\mathrm{m}$ sprint time was shown to be 16 weeks. In terms of program duration, Saez de Villarreal et al. (33) suggested a duration of less than 10 weeks (i.e., between 6 and 8 weeks) with 3-4 PT sessions per week using high intensities, which were found to be most beneficial to improve sprint performance. However, for an adequate training concept in soccer, 3-4 PT sessions per week in combination with competition games on weekends might be too much, and may likely overuse soccer players, resulting in fatigue and decreased performance because of a negatively influenced recovery period (9). For agility, we can conclude that HAR performance already had improved after only 4 weeks of PT; possible learning effects can be ruled out because of the fact that this test was used for almost 1.5 years in this academy as a standard agility test; therefore, all subjects were highly familiar with this test. Furthermore, the findings revealed that the greatest improvement is observed after 12 weeks of PT, with no change in performance during the last 4 weeks of PT. In summary, our data indicate that an adequate PT program duration is highly dependent on what type of explosive action (straight line sprint vs. agility vs. jumping performance) should be improved, as the time course of improvement is revealed to be very different in each parameter.

This study is also the first to use young elite Ghanaian soccer players as participants. Our participants already showed, considering their bone age (CG: $13.1 \pm 0.9$ years vs. PTG: $14.0 \pm 1.3$ years), total testosterone values (CG: $404 \pm 90 \mathrm{ng} \cdot \mathrm{dl}^{-1}$ vs. PTG: $\left.445 \pm 58 \mathrm{ng} \cdot \mathrm{dl}^{-1}\right)$ at the upper limit of reference values from Nichols Institute (25) for 12.0 13.9-year-old boys $\left(\leq 420 \mathrm{ng} \cdot \mathrm{dl}^{-1}\right)$, and were, therefore, mature enough to increase muscle mass. In general, bone age in both groups was almost 1 year higher than the chronological age but was not correlated. Maturity status, displayed by bone age, was related to all sprint and jump tests, but demonstrated no association with the 2 change of direction tests, HAR and MB5. This emphasizes that maturity effect's strength and speed abilities, whereas coordination and agility seem to be independent. Worth noting is that the relationships between chronological age and the above mentioned speed and sprint tests were lower, or not significant, demonstrating that maturity assessment is an important consideration in youth sports stratification. To the authors' knowledge, no specific reference data regarding testosterone or bone age values for Ghanaians is available. Finally, the recorded training volume of the Ghanaian soccer players was less when compared with other young elite soccer players (6.5 hours of training per week vs. 9 hours of training per week) (7).

\section{Practical Applications}

The 16-week PT applied in our study suggests that performing only 2 (of 8 ) slow SSC-type plyometric exercises per session is not sufficient to improve the initial acceleration phase. In contrast, 6 (of 8) fast SSC-type plyometric exercises seem to provoke significant improvement in the second acceleration phase and maximal velocity phase, respectively. In terms of agility, the applied PT is adequate to show improvements in general speed and jump performance but is lacking in the ability to improve change of direction performance incorporated in a soccer-specific speed and agility test. The strength and conditioning professional has to think about different methods to improve eccentric flexor strength, apart from our PT, to possibly further enhance specific speed and agility. Regarding recommendations about the program duration of a short-term PT program, it is highly dependent on what type of explosive action (straight line sprint vs. change of direction performance [agility] vs. jumping performance) should be improved. In our young elite athletes, it seems that 16 weeks of PT is enough to improve 20-m sprint performance, but after only 4 weeks of general agility and LJ performance show improvement, and after 8 weeks, MB5 is improved. Therefore, the strength and conditioning professional has to be very clear in their goal of training because our findings indicate that the time course of improvement of the single parameters is different.

\section{AcKnowledgments}

The authors thank the Red Bull Soccer Academy West Africa Ltd., especially Pieter Int'Groen and Thomas Harris for their support, as well as the U13 and U15 teams for their enthusiastic participation. Further, the authors thank Dr. Franz Sulzer, Michael Tschernkowitsch, Bettina Schauer, and Ursula Stadler. There were no professional relationships with companies or manufacturers. The results of the present study do not constitute endorsement of the product by the authors or the NSCA. Disclosure of funding received for this work from any of the following organizations: National Institutes of Health (NIH), Wellcome Trust, Howard Hughes Medical Institute (HHMI), and other(s).

\section{REFERENCES}

1. Baker, D. Improving vertical jump performance through general, special, and specific strength training. J Strength Cond Res 10: 131136, 1996

2. Bangsbo, J, Norregaard, L, and Thorso, F. Activity profile of competition soccer. Can J Sport Sci 16: 110-116, 1991.

3. Barnes, JL, Schilling, BK, Falvo, MJ, Weiss, LW, Creasy, AK, and Fry, AC. Relationship of jumping and agility performance in female volleyball athletes. J Strength Cond Res 21: 1192-1196, 2007. 
4. Bloomfield, J, Polman, R, and O'Donoghue, P. Physical demands of Different Positions in FA Premier League soccer. J Sports Sci Med 6: 63-70, 2007.

5. Bobbert, MF. Drop jumping as a training method for jumping ability. Sports Med 9: 7-22, 1990.

6. Bobbert, MF, Gerritsen, KGM, Litjens, MCA, and Van Soest, AJ. Why is countermovement jump height greater than squat jump height? Med Sci Sports Exerc 28: 1402-1412, 1996.

7. Buchheit, M, Mendez-Villanueva, A, Delhomel, G, Brughelli, M, and Ahmaidi, S. Improving repeated sprint ability in young elite soccer players: Repeated shuttle sprints vs. explosive strength training. $J$ Strength Cond Res 24: 2715-2722, 2010.

8. Christou, M, Smilios, I, Sotiropoulos, K, Volaklis, K, Pilianidis, T, and Tokmakidis, SP. Effects of resistance training on the physical capacities of adolescent soccer players. J Strength Cond Res 20: 783-791, 2006.

9. Chu, DA. Jumping into Plyometrics. Champaign, IL: Human Kinetics, 1998.

10. Cohen, J. The statistical power of abnormal-social psychological research: A review. J Abnorm Soc Psychol 65: 145-153, 1962.

11. Delecluse, C, Van Coppenolle, H, Willems, E, Van Leemputte, M, Diels, R, and Goris, M. Influence of high-resistance and high-velocity training on sprint performance. Med Sci Sports Exerc 27: 1203-1209, 1995

12. Diallo, O, Dore, E, Duche, P, and Van Praagh, E. Effects of plyometric training followed by a reduced training programme on physical performance in prepubescent soccer players. J Sports Med Phys Fitness 41: 342-348, 2001.

13. Ford, HT Jr, Puckett, JR, Drummond, JP, Sawyer, K, Gantt, K, and Fussell, C. Effects of three combinations of plyometric and weight training programs on selected physical fitness test items. Percept Mot Skills 56: 919-922, 1983.

14. Gonaus, C and Müller, E. Using physiological data to predict future career progression in 14- to 17-year-old Austrian soccer academy players. J Sports Sci 30: 1673-1682, 2012.

15. Gonaus, $\mathrm{C}$ and Müller, E. Test-retest reliability of a field-based fitness test battery used in Austrian youth soccer. In: 18th Annual Congress of the European College of Sport Science. Barcelona, Spain: Open Print 2013.

16. Graham-Smith, P and Pearson, SJ. An investigation into the determinants of agility performance. In: $3 r$ International Biomechanics of the Lower Limb in Health, Disease and Rehabilitation. Manchester, United Kingdom: The University of Salford, 2005.

17. Hakkinen, K, Alen, M, and Komi, PV. Changes in isometric forceand relaxation-time, electromyographic and muscle fibre characteristics of human skeletal muscle during strength training and detraining. Acta Physiol Scand 125: 573-585, 1985.

18. Harman, EA, Rosenstein, MT, Frykman, PN, and Rosenstein, RM. The effects of arms and countermovement on vertical jumping. Med Sci Sports Exerc 22: 825-833, 1990.

19. Ingle, L, Sleap, M, and Tolfrey, K. The effect of a complex training and detraining programme on selected strength and power variables in early pubertal boys. J Sports Sci 24: 987-997, 2006.

20. Jones, P, Bampouras, TM, and Marrin, K. An investigation into the physical determinants of change of direction speed. J Sports Med Phys Fitness 49: 97-104, 2009.

21. Kotzamanidis, C. Effect of plyometric training on running performance and vertical jumping in prepubertal boys. J Strength Cond Res 20: 441-445, 2006.

22. Meylan, C and Malatesta, D. Effects of in-season plyometric training within soccer practice on explosive actions of young players. J Strength Cond Res 23: 2605-2613, 2009.
23. Michailidis, Y, Fatouros, IG, Primpa, E, Michailidis, C, Avloniti, A, Chatzinikolaou, A, Barbero-Alvarez, JC, Tsoukas, D, Douroudos, II, Draganidis, D, Leontsini, D, Margonis, K, Berberidou, F, and Kambas, A. Plyometrics' trainability in preadolescent soccer athletes. J Strength Cond Res 27: 38-49, 2013.

24. Mohr, M, Krustrup, P, and Bangsbo, J. Match performance of highstandard soccer players with special reference to development of fatigue. J Sports Sci 21: 519-528, 2003.

25. Nichols Institute Related Guides: Testosterone, LC/MS/MS Available at: http://wwwquestdiagnosticscom/homehtml. Accessed December 4, 2013.

26. O'Donoghue, P. Time motion analysis of work rate in elite soccer. In: 4th World Congress of Notational Analysis of Sport. H.M.F. Tavaes, ed. Lisbon, Portugal: Faculty of Sport Sciences and Physical Education, University of Porto. 2001. pp. 65-70.

27. Plisk, SS. Speed, agility, and speed-endurance development. In: Essentials of Strength Training and Conditioning. T.R. Baechle and R.W. Earle, eds. Champaign, IL: Human Kinetics, 2008. pp. 458-485.

28. Potach, DH and Chu, DA. Plyometric training. In: Essentials of Strength Training and Conditioning. T.R. Baechle and R.W. Earle, eds. Champaign, IL: Human Kinetics, 2008. pp. 415-458.

29. Potach, DH, Katsavelis, D, Karst, GM, Latin, RW, and Stergiou, N. The effects of a plyometric training program on the latency time of the quadriceps femoris and gastrocnemius short-latency responses. J Sports Med Phys Fitness 49: 35-43, 2009.

30. Reilly, T, Bangsbo, J, and Franks, A. Anthropometric and physiological predispositions for elite soccer. J Sports Sci 18: 669-683, 2000.

31. Rubley, MD, Haase, AC, Holcomb, WR, Girouard, TJ, and Tandy, RD. The effect of plyometric training on power and kicking distance in female adolescent soccer players. $J$ Strength Cond Res 25: 129-134, 2011.

32. Rumpf, MC, Cronin, JB, Oliver, JL, and Hughes, M. Assessing youth sprint ability-methodological issues, reliability and performance data. Pediatr Exerc Sci 23: 442-467, 2011.

33. Saez de Villarreal, E, Requena, B, and Cronin, JB. The effects of plyometric training on sprint performance: A meta-analysis. $J$ Strength Cond Res 26: 575-584, 2012.

34. Saez-Saez de Villarreal, E, Requena, B, and Newton, RU. Does plyometric training improve strength performance? A meta-analysis. J Sci Med Sport 13: 513-522, 2010.

35. Sedano, S, Matheu, A, Redondo, JC, and Cuadrado, G. Effects of plyometric training on explosive strength, acceleration capacity and kicking speed in young elite soccer players. J Sports Med Phys Fitness 51: 50-58, 2011.

36. Sheppard, JM and Young, WB. Agility literature review: Classifications, training and testing. J Sports Sci 24: 919-932, 2006.

37. Tanner, JM, Whithouse, RH, and Marshall, WA. Assessment of Skeletal Maturity and Prediction of Adult Height (TW2 Method). London, United Kingdom: Acamdic Press, 1975.

38. Thomas, K, French, D, and Hayes, PR. The effect of two plyometric training techniques on muscular power and agility in youth soccer players. J Strength Cond Res 23: 332-335, 2009.

39. Valquer, W, Barros, TL, and Sant'anna, M. High intensity motion pattern analyses of Brazilian elite soccer players. Presented at IV World Congress of Notational Analysis of Sport. Lisbon-Porto, 1998.

40. Verheijen, R. The complete handbook of conditioning for soccer. Mich Data Reproductions 1998, pp. 388.

41. Withers, RT. Match analyses of Australian professional soccer players. J Hum Mov Stud 8: 159-176, 1982. 